\title{
TEORIA DO BEM-ESTAR DAS CRIANÇAS
}

\author{
LEENA ALANEN \\ Professora do Departamento de Ciências da Educação \\ da Universidade de Jyväskylä - Finlândia \\ leena.alanen@edu.jyu.fi \\ Tradução: Elba Siqueira de Sá Barretto
}

\section{RESUMO}

O texto discute as contribuições dos novos Estudos sobre a Infância em face da importância que têm assumido as crianças e a infância nos processos de reconfiguração do bem-estar social nas sociedades ocidentais avançadas.

ASSISTÊNCIA À INFÂNCIA - CRIANÇAS - POLITICAS PÚBLICAS - ASSISTÊNCIA SOCIAL

\begin{abstract}
THE CHILD WELFARE THEORY. The text discusses the contributions of new Studies on Childhood, given the importance that children and childhood have assumed in the process of reconfiguring social welfare in advanced Western societies.

CHILDHOOD WELFARE - CHILDREN - PUBLIC POLICIES - SOCIAL ASSISTENCE
\end{abstract}

Na recente década, a situação das infâncias contemporâneas e o bem- estar das crianças tornaram-se uma preocupação constante no discurso público e em debates políticos por toda a Europa. Um dos motivos decorre da apreensão quanto ao rápido envelhecimento das sociedades ocidentais: as pessoas hoje vivem mais que as gerações anteriores, enquanto as crianças estão nascendo em números decrescentes. Isso gera forte apreensão quanto à adequação de futuros trabalhadores e cidadãos, tanto em termos quantitativos como qualitativos. A mesma preocupação é compartilhada pelos diagnósticos da "crise" dos Estados de bem-estar e pela necessidade de reformulá-los. Além disso, discute-se também a necessidade de profunda revisão dos modelos de produção de bem-estar social. O que os Estados precisam fazer agora é desenvolver novos modelos que promovam "investimentos" em pessoas (ou seja:

Este artigo foi publicado originalmente em inglês e sua referência completa é: Theoerizing children's welfare. In: WINTERSBERGER, H. et al. (Ed.) Childhood, generational order and the welfare state: exploring children's social and economic welfare, v. I. Odense: University Press of Southern Denmark, 2007. p.27-44. (Cost Al 9: children's welfare). 
em "capital humano") em vez de redistribuir dinheiro e benefícios. As crianças, em particular, são uma subpopulação estratégica em que se há de investir, e "estratégias de investimento social com foco na criança" deveriam ocupar lugar central nos novos programas propostos para ir "além do Estado de bem-estar" (Pierson, 1998; Bonoli, George, Taylor-Gooby, 2000; Esping-Andersen, 2002).

Essas discussões estão ocorrendo em fóruns de fomento a políticas, mas a pesquisa científica de natureza social sobre o bem-estar parece estar muito defasada. As crianças, especialmente, nunca estiveram presentes no centro da pesquisa do bem-estar. Agências de defesa da infância (como o Fundo das Nações Unidas para a Infância - Unicef) e iniciativas de direitos das crianças (como a dos grupos que se ocupam da pobreza infantil) lideraram a produção e distribuição de informações sobre o status das crianças no mundo e em diferentes países. Com o passar dos anos, muitas outras iniciativas têm sido tomadas para elaborar indicadores com vistas a medir o bem-estar das crianças, tanto em sistemas nacionais como entre países, sobretudo com o propósito de produzir informação e orientar a formulação de políticas, bem como testar o seu desempenho e, mais recentemente, fornecer dados confiáveis para extensos relatórios sociais sobre o status das crianças e suas condições de vida.

Em cada caso, a questão sobre o que realmente significa o bem-estar das crianças recebe algum tipo de resposta, embora frequentemente ela permaneça implícita e, muitas vezes, repouse sobre "verdades" publicamente aceitas sobre o assunto. No mundo da formulação de políticas [policy making], isso talvez seja de se esperar, já que as racionalidades da política e da ciência tendem a não coincidir (Hudson, Lowe, 2004). Até o momento, parece que a base teórica dos esforços para promover o bem-estar das crianças esteve em segundo plano, no que se refere ao objetivo mais imediato de elaboração de protocolos comuns, de medidas compartilhadas e consistentes e de indicadores sintéticos do bem-estar infantil (Hauser, Brown, Prosser, 1997; Gasper, 2004; Manderson, 2005). No entanto, agora que as crianças e a infância estão se tornando um assunto mais proeminente na repaginação e reorganização do bem-estar social, a contribuição das Ciências Sociais está sendo solicitada. Em resposta a esse chamado, os "novos" Estudos sobre a Infância oferecem contribuições importantes.

Este artigo objetiva esclarecer algumas questões de base, buscando respostas da perspectiva dos Estudos sobre a Infância. $\bigcirc$ ponto de partida é dado 
pela revisão e discussão da experiência e dos resultados do projeto Cost A / 9: bem-estar das crianças'. Como deveríamos pensar o bem-estar no caso das crianças? Como deveríamos prosseguir com vistas a uma compreensão sociológica de seu bem-estar? $\bigcirc$ artigo examina o extenso e confuso acervo de diferentes noções de bem-estar localizadas na bibliografia sobre o tema, uma vez que esse é um dos conceitos-chave de qualquer Sociologia na elaboração do bem-estar da criança. Perguntamos então como as crianças e a infância são tratadas nesses estudos: elas estão presentes na pesquisa do bem-estar? E, se estão, de que maneira?

Com base nessa leitura, a sugestão é que, para progredir no entendimento do bem-estar das crianças, é necessário que haja um encontro entre os Estudos sobre a Infância e os Estudos sobre o Bem-estar. As formas contemporâneas de pensamento sobre o bem-estar são diretamente aplicáveis a grupos e categorias sobre os quais o bem-estar ainda não tenha sido considerado? Podemos simplesmente "adicionar" as crianças ao referencial dos estudos de bem-estar? As experiências adquiridas no desenvolvimento dos Estudos Sociais das crianças e sobre a infância durante as duas últimas décadas geraram algumas dúvidas sobre o sucesso de uma estratégia adicional. Elas nos informam que teorias e conceitos, metodologias e medidas da ciência social moderna e clássica foram principalmente interpretados a partir de um ponto de vista normativo adulto ${ }^{2}$. A exclusão das crianças de suas preocupações foi a regra. Portanto, para tornar as ferramentas e o conhecimento da ciência social significativos no caso de crianças e da infância, ao mesmo tempo em que nos esforçamos para evitar sua distorção "adultista", será necessário mais do que apenas uma adição: primeiro, uma crítica da pesquisa de bem-estar existente e uma desconstrução de suas formas de pensamento, ambas interpretadas do ponto de vista explícito das crianças. Sugere-se que algumas das ferramentas

I. COST A 19: children's welfare é um projeto realizado no contexto da European Cooperation in the Field of Scientific and Technical Research, entidade que se propõe a fortalecer a rede de pesquisadores da Comunidade Europeia em torno de nove campos de conhecimento. Iniciado em 200 I, e envolvendo diversos países da Comunidade Europeia, o projeto se propôs a analisar o bem-estar das crianças em sociedades envelhecidas [ageing societies]. Os estudos deram origem a publicações e conferências. Para mais informações acionar: http://www.svt.ntnu.no/noseb/costal9/ (N. da E.)

2. Para adultism em ciência social, ver Alanen (1992). 
analíticas desenvolvidas nos Estudos sobre a Infância podem ser usadas proficuamente no exame da utilidade das estruturas de bem-estar existentes para uma Sociologia do Bem-Estar das Crianças.

\section{DESLINDANDO O BEM-ESTAR: DISTINÇÕES ÚTEIS}

Para sustentar esses argumentos, utilizamos uma distinção que Ruth Lister (2004) desenvolve na leitura que faz das pesquisas sobre pobreza. $\bigcirc$ foco de suas considerações críticas é o balanço dessas pesquisas, especialmente a diversidade de maneiras pelas quais os pesquisadores têm lidado com o termo pobreza. Na literatura, muitos usos diferentes do mesmo termo podem ser encontrados, os quais conduzem também à elaboração de políticas. Lister assinala que, para entender o fenômeno da pobreza, é importante diferenciar entre conceitos, definições e medidas (Lister, 2004, p.3-8). Conceitos operam em um nível bem geral e fornecem a estrutura dentro da qual definições (de conceitos) e medidas (operacionalizações de definições) são desenvolvidas. Definições (e, portanto, também medidas) permeiam conceitos e, nesse sentido, nas definições de pobreza estão, na verdade, implícitas explicações sobre a pobreza e sua distribuição. Por isso, é importante, primeiro: que as definições não estejam divorciadas de suas conceituações mais abrangentes, e, segundo: que suas relações com os referenciais conceituais mais abrangentes (e podemos visualizar esses referenciais como redes de conceitos inter-relacionados) sejam esclarecidas. Somente então, afirma a autora, as definições podem funcionar como uma base adequada para medidas de desenvolvimento.

O problema em muitas pesquisas sobre o tema, observa Lister (2004, p.6-7), é que os pesquisadores costumam iniciar seu trabalho com definições de pobreza e continuam com o desenvolvimento de medidas de pobreza mas, no processo, eles tendem a confundir a sua definição do conceito, ou simplesmente fundir definições e conceitos. O resultado é que se perdem de vista, na análise, os referenciais em que se assentam diferentes entendimentos de pobreza, e a interpretação histórica e política da noção de pobreza adotada deixa a desejar. Isso, é claro, tem implicações cruciais para a política voltada à pobreza, na medida em que conceitos não se sustentam (ou são contestados) fora da história e da cultura e são contestados, tendo efeitos práticos. 
Do mesmo modo, a confusa noção de bem-estar (das crianças) precisa ser levada tão a sério quanto a de "pobreza" na pesquisa sobre pobreza: a teoria do bem-estar das crianças exige que o bem-estar seja adequadamente conceituado e as referências em que se baseiam os conceitos de bem-estar das crianças sejam explicitadas.

Uma outra diretriz para se pensar em como teorizar o bem-estar das crianças é fornecida por O'Brien e Penna que, em sua discussão geral sobre a teoria do bem-estar, fazem distinção entre dois de seus componentes interligados: uma teoria do bem-estar social e uma teoria social do bem-estar. Ao teorizar sobre o bem-estar social, o foco incide sobre

...como a distribuição de recursos e oportunidades e como os padrões sociais de acesso, participação, inclusão e exclusão apoiam ou contribuem para minar o bem-estar individual e coletivo. Resumidamente, trata-se de uma teoria da organização das relações sociais e dos impactos dessa organização no bem-estar individual e coletivo. (1998, p.4)

Essa maneira de teorizar é essencialmente uma teoria para a política de bem-estar, já que tenta (teoricamente) alargar visões particulares da vida social e política implícitas na política e nos referenciais de bem-estar que pretendem gerar ações e resultados políticos mais "eficazes", "racionais", "justos", "humanitários", ou seja, mais "apropriados". Suas premissas e pressupostos básicos recaem normalmente sobre o comportamento das pessoas, que aprendem ou interagem, sobre a formação e a modificação de suas atitudes, ou sobre como as pessoas geram recursos e os compartilham. Essas são as premissas dos referenciais de bem-estar que orientam a elaboração de políticas sociais e os programas de bem-estar, bem como a sua implementação; e elas nos dirão como o bem-estar será oferecido e como a vida será levada quando a política ou o programa for implementado (O'Brian, Penna, 1998, p.4-5).

O'Brien e Penna afirmam que esse tipo de teorização é essencialmente normativo: ele nos diz como achamos que as pessoas deveriam agir e que crenças elas deveriam ter, como instituições políticas ou econômicas deveriam operar e como as organizações deveriam se relacionar com as populações a que servem se fizéssemos as políticas e programas funcionar "melhor", ou seja, produzir a pretendida eficiência ou os resultados esperados. 
Não é necessário de modo algum atribuir um sentido negativo a uma teoria com essa lógica normativa, como se se referisse necessariamente à manipulação e à direção das crenças e dos comportamentos de pessoas. $\mathrm{Na}$ verdade, muitas vertentes históricas e recentes da teoria política e da crítica social - como as das teorias liberais, marxistas ou ecológicas - mostram grande preocupação com a mudança dos comportamentos das pessoas ou instituições sociais com vistas a criar estruturas e relações mais justas, igualitárias ou democráticas, e com isso, o bem-estar, não importa a maneira como sejam definidos em cada caso. A normatividade na teorização do bem-estar social nos alerta para a importância do reconhecimento dos critérios de bem-estar que de fato utilizamos quando especificamos o que queremos dizer com o bem-estar das crianças. Na elaboração de políticas, eles permanecem, na maioria das vezes, implícitos, ao passo que a Sociologia do Bem-Estar (das crianças) precisaria ser clara acerca dos critérios normativos do bem-estar utilizados em cada caso.

O segundo componente de uma teoria do bem-estar é o que focaliza "como as políticas sociais e os programas de bem-estar emergiram e como adquiriram a forma que têm, e ainda que relações existem entre as políticas e programas e as sociedades que os adotam" (O’Brien, Penna, 1998, p.6).

A premissa para o desenvolvimento desse tipo de teoria social de bem-estar é a percepção (sociológica) de que as políticas do bem-estar, programas e instituições - e o Estado de bem-estar como um aparato dessas políticas, programas e instituições - não apenas distribuem e redistribuem rendimento e outros benefícios às pessoas, mesmo quando essa é a intenção benigna das políticas. Tais políticas também têm impacto nas relações sociais entre as pessoas e às vezes de forma muito poderosa, incluindo ou excluindo, trazendo algumas para o centro do reconhecimento e marginalizando outras, agindo para a liberação de umas e, talvez, para a opressão de outras. John Clarke (2004, p.9), por exemplo, ao escrever sobre o desenvolvimento do Estado do bem-estar no ocidente nos anos 60 e 70, afirma que ele não representava simplesmente o aumento de um campo previamente determinado de benefícios e serviços; era marcado também por uma série de lutas fragmentadas e sobrepostas que produziam diferenças, divisões e formas de desigualdade, baseadas nas demandas sociais desses Estados. 
A teoria do bem-estar nesse sentido do termo envolve teorização das lutas políticas e culturais sobre o bem-estar, os agentes ativos nessas lutas e a criação de relações sociais e divisões delas resultantes. Esses dois componentes da teorização do bem-estar não são formas alternativas de se entender o bem-estar; ou que competem entre si, ou melhor, juntos eles delineiam um programa de pesquisa abrangente com vários níveis e dimensões. A tarefa de qualquer projeto de pesquisa é mais modesta do que a de um amplo programa de pesquisa.

\section{O BEM-ESTAR DAS CRIANÇAS E O PROJETO COST}

Os objetivos do projeto COST foram apresentados em seu plano de trabalho (Memorando de Acordo - MoU, 2000) de maneira bem vaga: não especificavam o que exatamente o projeto precisaria examinar e como, para poder explicar o bem-estar das crianças em vários países europeus. $\bigcirc$ projeto tinha como objetivo:

- melhor compreender o bem-estar das crianças sob o impacto da globalização, internacionalização e do mercado, como fatores crescentes nas suas vidas;

- melhor compreender as diferenças quanto ao bem-estar, espaço e uso do tempo entre crianças e entre grupos de gerações (exemplo crianças versus adultos);

- melhor compreender o provimento às crianças por meio dos orçamentos públicos;

- conferir transparência ao atendimento das crianças no que diz respeito à distribuição de recursos entre as gerações e ao uso do espaço e do tempo pelas crianças.

A motivação básica para o lançamento do projeto partiu do reconhecimento de que as crianças e a infância não foram amplamente consideradas na pesquisa de bem-estar europeia e na discussão sobre a política de bem-estar. projeto propôs-se, portanto, a dar mais proeminência às crianças e à infância no discurso do bem-estar, e a começar a produzir conhecimentos que sejam também relevantes para a política europeia para a criança, embora não 
estejam limitados a ela. Isso seria atingido baseando a pesquisa nos princípios e no desenvolvimento dos "novos" Estudos Sociais sobre as crianças e a infância (Estudos sobre a Infância). A abordagem adotada na coleta, utilização e interpretação de dados secundários nacionais foi de caráter geracional (Jensen et al., 2004, Introdução).

Dois "pilares" do bem-estar foram especificados inicialmente no MoU para conduzir o projeto a um entendimento mais aprofundado sobre o bem-estar das crianças. Primeiro, o bem-estar social e econômico das crianças seria estudado e, segundo, seriam analisados o acesso ao espaço e a utilização do tempo feita por elas. Esses dois "pilares" também serviram como diretrizes gerais aos pesquisadores que compilaram os estudos dos respectivos países participantes visando relatar como se dá o bem-estar das crianças em cada um deles $^{3}$. Dois grupos de trabalho foram criados para se aprofundar especificamente em cada um dos "pilares do bem-estar", definindo assim o duplo foco do trabalho subsequente do projeto.

Inicialmente não foram oferecidas maiores orientações conceituais além dessas para o trabalho das equipes nacionais de pesquisa. Embora o projeto também não discutisse vários "assuntos de bem-estar", ou "dimensões" (particularmente a pobreza infantil), ele não comprometia os participantes com nenhuma tradição particular ou abordagem na área de pesquisa sobre o bem-estar. Esse também parecia um ponto de partida muito razoável, considerando o método de trabalho adotado (análise interpretativa de dados secundários) e a grande diversidade, seja da quantidade, seja do tipo de dados relacionados à infância, disponível nos respectivos países. Teve igualmente importância o curriculum profissional extremamente diversificado dos pesquisadores, que, embora experientes como cientistas sociais, pertenciam a tradições disciplinares diferentes, o que se refletiria nos relatórios de cada país e nos outros produtos do projeto. Por fim, o entendimento de "bem-estar" adotado individualmente pelos pesquisadores pode ser dificilmente separado dos contextos políticos e culturais de seu trabalho nos próprios contextos nacionais, e, portanto, seria de se esperar que o relatório nacional sobre o bem-estar das crianças também refletisse significados e entendimentos locais.

3. Ver os estudos dos 13 países publicados em Jensen et al. (2004). 


\section{SIGNIFICADOS DE BEM-ESTAR}

A ideia e o conceito de bem-estar são, obviamente, muito mais antigos que a pesquisa sobre o bem-estar como um campo de trabalho científico, ou que a política de bem-estar como um domínio da práxis social. Desde sua origem na filosofia política (Barry, 1999), diversos significados, antigos e novos, foram adicionados e tirados da noção. Concepções universais de bem-estar humano têm uma longa história e são agora parte do discurso internacional, conforme exemplificado pelos Relatórios de Desenvolvimento Humano, do Programa de Desenvolvimento das Nações Unidas (Clark, Gough, 2005). Hoje a filosofia política e a economia moderna - dois discursos diferentes - são as principais produtoras no campo de Estudos de Bem-Estar (Gasper, 2004). Com a evolução e o crescimento, particularmente na última metade do século 20 , do aparato social, político e administrativo que hoje conhecemos como Estado de bem-estar, os usos do termo se multiplicaram e novos subcampos foram abertos na pesquisa do bem-estar. A análise da política social é atualmente uma (terceira) voz forte no estudo do tema. A preocupação política em torno da "crise" do Estado do bem-estar, especialmente durante os anos 90, e a busca de uma nova "arquitetura do bem-estar", também contribuíram para a proliferação dos significados do termo.

A multiplicidade de noções de bem-estar hoje existente e o problema de entender como elas se diferenciam e se relacionam entre si torna-se evidente quando se examina até mesmo uma pequena parte da atual produção bibliográfica das pesquisas sobre o tema. A seguinte amostra, não exaustiva, retirada da vasta literatura das ciências sociais, sempre em crescimento, é suficiente para demonstrar isso ${ }^{4}$.

"Bem-estar", foi-nos dito, pode significar:

- riqueza, saúde, alegria [eudaimonia], ou "ventura" de uma pessoa ou um grupo;

4. Os exemplos são tomados de Froste Stein (1989), Carrington(1993), Bilton (1999), Chamberlayne et al. ( 1999), Berger-Schmitt (2000), Berger-Schmitte Noll (2000), Falkingham (200 I), Vleminckx e Smeeding (200 I), Goldson, Lavalette e Mckechnie (2002); Hendrick (2003), Laderchi, Saith e Stewart (2003), Gasper (2004). 
- um bem, ou seja, uma preferência individual, como na economia do bem-estar;

- qualidade de vida cotidiana, de indivíduos ou grupos;

- desenvolvimento humano.

Essas definições de bem-estar apontam para avaliações qualitativas da situação material, física ou social de indivíduos ou grupos, e, portanto, referem-se basicamente ao estado de indivíduos que estão bem [being well], estão passando bem [doing well] ou passam bem [faring well]. Em muitas pesquisas sobre o bem-estar e na literatura política, o well being e o welfare são tratados como sinônimos de bem-estar, conforto, felicidade (Gasper, 2004, p.2-3). Particularmente nas tradições de medição quantitativa de bem-estar individual, o welfare parece ser o termo mais utilizado (Rapley, 2003, p.26-62).

O significado real de estar bem, estar passando bem, ou passar bem em cada caso é um assunto normativo, decidido separadamente de suas medições (Corbett, 1997). Indicadores sociais são particularmente desenvolvidos como instrumentos para teste, gerenciamento e governança de políticas sociais; com o auxílio de indicadores, uma nova política pode ser testada para se verificar o que ela produz em relação ao grupo social visado (Ben-Arieh, Wintersberger, 1997; Corbett, 1997). O aspecto principal está no desempenho da política e não nos processos pelos quais o produto final da política é gerado (Corbett, 1997, p. 19), assim os indicadores sociais são considerados ferramentas para medir o resultado da política, como, por exemplo, o status de crianças/da infância. Desse modo, um dos significados principais de bem-estar das crianças é o status das crianças individuais em relação a alguma condição definida da situação das crianças, por exemplo, a saúde, a educação, a segurança econômica ou um comportamento problema.

Indicadores de bem-estar das crianças neste sentido foram desenvolvidos há mais de duas décadas pelo Unicef, a fim de monitorar regularmente a situação das crianças do mundo. Um elemento mais novo em diversos países

5. A esse respeito, Gasper (2004, p.22) observa que etimologicamente bem-estar poderia ter uma acepção muito mais ampla do que sentir-se bem, já que "fare [da palavra em inglês para bem-estar: welfare] significa viajar, passar pela vida, em vez de estar somente em um momento". 
europeus é a inclusão de sistemas semelhantes de medição do bem-estar das crianças em relatórios sociais 6 .

Um segundo conjunto de concepções de "bem-estar", parcialmente diferente e mais abrangente, inclui:

- assistência financeira e de outras formas, dada às pessoas pobres;

- oferecimento organizado de assistência educacional, cultural, médica e financeira aos necessitados;

- sistemas de provisão de serviços cujo objetivo explícito é promover o bem-estar social e aliviar o sofrimento social,

- procedimentos estatutários desenhados para promover o bem-estar físico e material das pessoas.

Esses usos mais "sistêmicos" do termo têm aumentado a partir da evolução e do crescimento dos Estados de bem-estar. "Bem-estar" passa a dar nome aos diversos sistemas de benefícios e serviços, até agora nacionais, oferecidos pelo Estado (de bem-estar). A literatura britânica usa particularmente o termo "bem-estar da criança" (e não "bem-estar das crianças") para designar áreas de política específicas voltadas ao oferecimento dos benefícios, em um dos sentidos mencionados, a todas as crianças da nação ou a várias categorias delas (exemplo, "crianças sob cuidados"). Alternativamente a essa literatura, "o bem-estar da criança" funciona como uma abreviatura referente ao aparato social, político e administrativo institucionalizado para esse fornecimento (Frost, Stein, 1989; Stevenson, 1999; Hendrick, 2003, 2005).

Na literatura norte-americana, o uso sistêmico não é muito claro, e "bem-estar" é empregado com um sentido muito mais restrito que na Europa. O termo aparece mais no contexto de programas específicos de assistência pública (como em "reforma do bem-estar") ou para referir-se à condição de estigmatização/estigmatizada de ser dependente desses programas, como "estar

6. Ver Joos (200I), para o caso da Alemanha, onde o relato social sobre as crianças é visto como um instrumento para desenvolvimento e avaliação do desempenho da política da infância do país [Politik für Kinder]. Para a variedade de domínios em que o bem-estar das crianças é medido, no caso do Reino Unido, ver Micklewright e Stewart (2000), e para o caso norte-americano, Hauser, Brown e Prosser (1997). 
no bem-estar"; "dependência de bem-estar"; "mães de bem-estar" (Currie, 1995; Barry, 1999, p. I26; Bloch et al., 2003; Clarke, 2004, p.21-22). O uso mais limitado semanticamente pode derivar do escopo mais limitado do sistema de bem-estar norte-americano em comparação com as estruturas do Estado de bem-estar europeu?

O tratamento sociológico do uso sistêmico de "bem-estar" assim descrito pode-se expandir para abranger a totalidade do sistema de bem-estar do Estado (e mesmo ir além dele): o bem-estar pode ser geralmente usado para se referir a todas as instituições, políticas e práticas de Estados de bem-estar e se pode ainda falar de culturas de bem-estar diferentes (Chamberlayne et al., 1999). Esse sentido abrangente de bem-estar também cobre os atores que fazem parte do e mantêm o sistema de instituições, políticas e práticas: gestores, administradores, prestadores de serviços e "clientes" ou "sujeitos" de bem-estar ${ }^{8}$, bem como as relações entre eles.

De acordo com a compreensão mais complexa e sistêmica de bem-estar, as noções particulares apresentadas podem agora fazer referência a resultados de atividades de bem-estar organizadas, a práticas e relacionamentos que fazem parte do sistema de bem-estar no âmbito de seus "usuários finais", que são cidadãos individuais e grupos deles. A ideia da produção de "bem-estar" impõe ainda que não só os representantes do Estado, mas também todos os outros representantes que participam da produção de bem-estar estejam incluídos

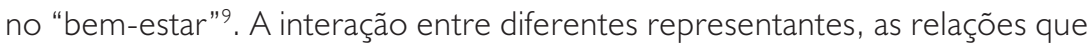
se criam entre atores, os resultados no nível individual e as divisões sociais recriadas na interação são elementos importantes no conjunto do bem-estar. Explicar esse sistema de bem-estar dinâmico com múltiplos níveis e múltiplos

7. Clarke (2004) observa o trabalho cultural intenso e sistemático nos Estado Unidos nos anos 80 e 90 para reconstruir o significado de "bem-estar". Fruto de uma separação política e institucional entre a segurança social (baseada na seguridade social) e a assistência condicional (baseada no exame da carência de recursos), o bem-estar foi criado para se referir a um programa de assistência pública específico (AFDC); esse foi interpretado "sistematicamente como o significado hegemônico de bem-estar" (p.22).

8. O’Brien e Penna ( 1998 ) sobre a "teorização do bem-estar social".

9. Os diversos centros e agentes de produção de bem-estar estão focalizados em discussões políticas e científicas de modelos de produção de bem-estar: eles incluem "regimes de bem-estar" (Esping-Andersen, 1990), "mix do bem-estar mix" ou "pluralismo do bem-estar" (Evers, Olk, 1996) e "triângulo do bem-estar" ou "quadrado do bem-estar" (Trifiletti, I 999). 
representantes, seja quantitativa, qualitativamente ou em ambos os aspectos, torna-se uma tarefa para pesquisa, a qual pode ser abordada de muitos pontos de vista diferentes.

Esses dois significados parecem ser os usos principais do termo "bem-estar"; ambos também podem ser relacionados ao bem-estar de crianças (assim como de outras subpopulações). Além deles, um terceiro e diferente uso também está surgindo no discurso do bem-estar, ligado particularmente a preocupações macroeconômicas e ambientais. Dentre essas noções mais novas estão a sustentabilidade, a "tolerabilidade" ou a coesão social de uma sociedade (Berger-Schmitt, 2000); sua atenção está direcionada à qualidade social e econômica de sociedades inteiras e populações como um todo.

\section{AS CRIANÇAS NAS PESQUISAS SOBRE O BEM-ESTAR: ALGUMAS OBSERVAÇÕES}

A motivação básica para o lançamento do projeto COST foi, como mencionado, o reconhecimento de que as crianças e a infância ainda não foram consideradas na pesquisa europeia sobre o bem-estar e no trabalho de política do bem-estar. Examinando a literatura da pesquisa de bem-estar, a observação geral confirma o seguinte: os assuntos de bem-estar das crianças estão totalmente ausentes ou são considerados somente de maneira indireta, por se relacionarem a algum outro tema (principal), como problemas de horário de trabalho dos pais e necessidades de cuidado das crianças em horário de trabalho.

Nos casos em que as crianças são expressamente incluídas, o tratamento tende a ser o de um único problema por vez: pobreza, saúde, mortalidade, negligência, crime, abuso, exclusão da educação e, mesmo, satisfação na vida (Micklewright, Stewart, 2000); sob o tema do bem-estar das crianças, o que se discute habitualmente é sua pobreza, saúde ou outros problemas de bem-estar. Raramente o bem-estar das crianças está posto como tal em foco. Nesses raros casos, ele costuma ser discutido mediante a "instrumentalização" das crianças. O recente discurso das políticas que promovem "estratégias de investimento social centralizadas na criança" (Esping-Andersen, 2002, numa perspectiva crítica; Beauvais, Jenson, 2001 ; Lister, 2002) é um exemplo: as crianças são vistas e avaliadas como trabalhadores e cidadãos do futuro; elas não aparecem 
como o que são ou como seu bem-estar pode ser considerado hoje. Atenção é dirigida às crianças principalmente porque se espera que o seu valor social se materialize no futuro (na idade adulta), e, portanto, elas são apresentadas como objetos racionais de "investimentos" econômicos, sociais ou culturais (educacionais) hoje - crianças são instrumentalizadas para o melhor da sociedade futura. Investimentos são propostos na forma de alívio à pobreza infantil, oferecimento de benefícios a famílias com crianças ou serviços de creche de boa qualidade. Esses são "investimentos" no sentido de que se pode esperar que deles haja retornos sociais em algum momento no futuro. E as crianças contam, porque elas são recursos da sociedade - instrumentos - para um futuro bem-estar melhor ${ }^{10}$.

O sociólogo reconhecerá facilmente atuando aqui uma ou outra ideia convencional de "socialização": a base de compreensão da natureza social das crianças é o pressuposto, não questionado, de que elas são pessoas ainda em processo de transformação (James, Jenks, Prout, 1998; Alanen, 1992). Elas, portanto, são contadas principalmente como futuros adultos (trabalhadores, pessoas físicas, cidadãos), não como crianças de hoje.

Os "novos" Estudos Sociais sobre a Infância desenvolveram por algum tempo o ponto de vista alternativo de que as crianças existem também como seres sociais que vivem suas vidas cotidianas e seus relacionamentos nas sociedades presentes. As crianças também são atores sociais e participantes da rede de relações que formam a sociedade; por meio de sua participação, elas também contribuem para as sociedades a que pertencem e não somente o fazem na idade adulta.

Essas novas perspectivas sobre as crianças e a infância, ou os resultados dos estudos sobre a infância, parecem não ter tido impacto nos discursos de bem-estar da criança até o momento (Fink, Lewis, Clarke, 200 I, p. I I 3, p. I 64). Janet Fink nota que:

10. Essa ideia de investimento em crianças tem, nos últimos anos, carreado muita simpatia e interesse nas comunidades de policy-making europeias (como na Comissão da União Europeia e na Organização para Cooperação e Desenvolvimento Econômico - OCDE), e é cada vez mais proposta como um modelo para a reconstrução dos Estados de bem-estar europeus (Bonoli, George, Taylor-Gooby, 2000, p. I19-138). 
... infância e crianças são altamente invisíveis em trabalhos comparativos [sobre a política de bem-estar europeia], com sua dupla ênfase na origem e no desenvolvimento de Estados de bem-estar e na medição do desempenho da seguridade social e do provimento. Extensas pesquisas foram realizadas sobre provimento às crianças pequenas na Europa [...] mas abordagens mais analíticas dos relacionamentos entre a criança, sua família e o Estado tendem a não estar integradas à política social convencional e à política social comparada... ou a ser marginalizadas como "Estudos sobre a Infância”... (200 I , p. 172)

Em Crianças, bem-estar e o Estado, Goldson, Lavalette e Mckechnie (2002) fazem uma tentativa louvável nessa direção. Os primeiros capítulos apresentam a "nova Sociologia da Infância" e a importância de "ouvir as vozes das crianças" (p.42-58); no entanto, o restante dos capítulos segue a tradição de discutir um tema de bem-estar por vez (pobreza, educação, trabalho, crime, abuso...) sem relacioná-lo às novas perspectivas sobre a infância.

Isso aponta para a relativa inutilidade da estratégia de "adicionar as crianças à ciência", que é uma repetição do primeiro esforço de trazer as mulheres e suas vidas e experiências para as ciências sociais nos anos 70 (Harding, 1986; para considerações paralelas sobre pesquisa acerca da infância, ver Alanen, 1992). Descobriu-se que essa não era uma abordagem adequada para as preocupações das mulheres (e feministas); também são necessárias abordagens mais integrativas para a inclusão das crianças na pesquisa sobre bem-estar.

Janet Fink (200I, p.173-174) caminha um pouco nessa direção em seu tratamento da política familiar. Ela acredita que o problema das crianças é realmente significativo para a análise da política, e de três maneiras. A primeira maneira é que a invisibilidade das crianças sustenta a permanência do desconhecimento dos modos pelos quais mudanças nas ideias sobre infância impactam e orientam as políticas para a família. A segunda é que a consideração das crianças como dependentes tem efeitos na construção da "parentalidade" (parenthood) com seus direitos e responsabilidades. A terceira é que a "familiarização" das crianças faz que elas desapareçam dentro da família, o que, por sua vez, ajuda a manter a crença de que as necessidades das crianças são satisfeitas quando se atendem as necessidades das famílias. A autora conclui: "a atenção à natureza desses discursos [sobre infância] [...] colocaria a criança em 
primeiro plano, como sujeito, nas políticas e práticas de bem-estar, de modo que a 'caixa-preta' da família, no contexto da política social comparada, seja aberta" (Fink, 200 I, p. 174).

Essa proposta de abordagem respeita a ação das crianças [children's agency] e está de acordo com os princípios básicos dos Estudos sobre a Infância.

Passando à outra área de pesquisa sobre bem-estar, embora a infância constitua uma criação da modernidade e a sua instituição seja fortemente mantida pelo Estado de bem-estar, a pesquisa do Estado de bem-estar estranhamente "esqueceu" as crianças e a infância. Na introdução de um livro sobre crianças e o Estado de bem-estar, Renate Kränzl-Nagl, Johanna Mierendorff e Thomas Olk (2003) explicam por que isso ocorre: o Estado de bem-estar é uma interpretação "adultista", criada por meio de lutas em torno das preocupações adultas, e as políticas do Estado de bem-estar são políticas para adultos. As políticas da infância [Kinderpolitik] começam a aparecer no discurso inicialmente com a observação de que as políticas sociais e econômicas, ou seja, as políticas para adultos, têm impacto também sobre crianças. Na verdade, a ausência de uma política para a infância bem desenvolvida por essa via cria, indiretamente, no discurso público o reconhecimento de que o Estado de bem-estar precisa de uma política explícita para a infância.

\section{ESTUDOS SOBRE A INFÂNCIA E ESTUDOS SOBRE O BEM-ESTAR: UM ENCONTRO PRODUTIVO}

As ambições do projeto Cost baseiam-se em uma visão das crianças e da infância que se desenvolveu desde os anos 80 por meio de diversas ciências sociais. Embora os Estudos sobre a Infância não constituam uma escola coerente de pensamento científico e não disponham de um paradigma homogêneo, eles adotam um número de pressupostos sobre as crianças e a infância amplamente compartilhados.

Um desses pressupostos muito enfatizados é o de que as crianças também são "atores sociais" e participantes ativos com contribuições para a vida cotidiana das sociedades. A invisibilidade, de longa data, das crianças na maioria das pesquisas das ciências sociais é vista como ligada a várias concepções de desenvolvimento e de socialização, que apresentam as crianças como se elas 
estivessem em diversos processos concomitantes de vir a ser. $\bigcirc$ ponto de partida contrastante do pressuposto da ação (social) das crianças implica, para a pesquisa, que elas são tratadas como sociologicamente iguais aos adultos ou a qualquer outra categoria social de pessoas.

O segundo pressuposto básico importante nos Estudos sobre a Infância diz respeito à instituição da infância: trata-se de uma formação "construída" histórica, social e politicamente, que foi institucionalizada para os membros mais jovens da sociedade. Infâncias sociais (e não somente infâncias individuais), portanto, são muitas; elas variam no tempo e no espaço, e supor que exista uma infância universal e "normal" é resvalar em uma ficção modernista. ○ que esses princípios básicos implicam para disciplinas como a Sociologia, Antropologia, Economia, Política Social, História etc. varia: para sociólogos que pesquisam sobre a infância, o desenvolvimento pode ser descrito em termos de três abordagens de trabalho diferentes" .

Uma Sociologia das crianças tem-se desenvolvido a partir da crítica à invisibilidade das crianças no conhecimento produzido pelas ciências sociais e da subsequente correção da abordagem de pesquisa para incluir as crianças. Nos novos estudos, as crianças têm sido postas no centro das atenções sociológicas e estudadas em razão de seus direitos próprios e não como apêndices ou anexos do restante do mundo social. $\bigcirc$ fim da discriminação sociológica das crianças também requer que os pesquisadores incluam em seu material de investigação as visões, experiências, atividades, os relacionamentos e conhecimentos das crianças, diretamente e em primeira mão. As crianças agora tomadas como unidades de pesquisa, e vistas como atores sociais e participantes do mundo social cotidiano, são consideradas como contribuindo com os eventos e assim também reproduzindo e transformando o seu mundo social. Métodos de pesquisa qualitativa, particularmente a etnográfica, são preferidos nas novas linhas de pesquisas sobre a infância'2.

I I. As "três sociologias" (Alanen, 200 la) devem ser entendidas aqui como diferentes métodos de fazer pesquisa sobre a infância com um referencial construcionista compartilhado. Cada uma delas tem um objetivo específico em termos do conhecimento que quer obter, bem como uma maneira específica de conceituar as infâncias; elas também utilizam métodos de pesquisa específicos.

12. Hoje essa é a abordagem dominante em termos quantitativos nos Estudos sobre a Infância. 
Uma segunda abordagem, uma Sociologia desconstrutivista (da noção) de crianças e das infâncias, surgiu de discussões das ciências sociais em torno das metodologias pós-positivistas e mais amplamente construcionistas, e suas implicações em relação ao modo como o mundo social deveria ser entendido e pesquisado. As noções de "criança", "crianças" e "infância" e suas muitas derivações foram vistas como interpretações culturais formadas historicamente. Essas noções são significativas para a realidade cotidiana das crianças quando incorporadas aos modelos sociais de ação, às práticas culturais e às políticas sociais, e elas oferecem scripts culturais e justificativas para que as pessoas ajam em relação às crianças e à infância. Discursos da infância derivam seu significado político fundamentalmente dessa perspectiva. Assim sendo, a tarefa de desconstrução do pesquisador é a de destrinçar essas interpretações, expondo seus criadores e as circunstâncias sociais em que foram geradas, bem como os processos políticos de sua produção, interpretação, comunicação e implementação prática. Essa sociologia busca divulgar o poder (discursivo) dos constructos culturais na vida social.

Há também uma terceira, a abordagem estrutural da infância. Nela a infância é tomada como unidade de análise e entendida como uma estrutura social. "Estrutura", no entanto, é um conceito com múltiplos significados, e há variedades de análises estruturais. Todas têm foco na macroanálise. Abordagens estruturais-categoriais implicam pensar as crianças como um agregado. "Elas permitem que descrevamos a população de crianças de acordo com um número de variáveis: é importante saber o que é comum para todas as crianças" (Qvortrup, 1993, p. 19). As crianças realmente vivas, cada uma com sua infância experienciada individualmente e de forma diferente - objeto de preocupação central da Sociologia das Crianças -, recebem agora pouca ou nenhuma atenção e são agrupadas na categoria crianças, estabelecida socialmente. A tarefa é relacionar observações empíricas no nível das vidas cotidianas das crianças (por exemplo, a experiência da pobreza, o uso do tempo, a exclusão social) a seus contextos no macronível. O propósito é identificar as estruturas sociais específicas e os processos em larga escala que interagem com outras estruturas e outros processos de impacto na vida cotidiana das crianças e nas suas condições de vida e "produzem" a pobreza, os padrões de uso do tempo ou a exclusão observados. Métodos quantitativos de pesquisa (estatísticos) são particularmente úteis, considerando as características sociais que as crianças compartilham e os seus contextos estruturais. 
Uma segunda abordagem estrutural adota o pensamento relacional: a infância é conceituada como uma posição gerada socialmente dentro de uma estrutura geracional, e definida como a configuração de relações geracionais específicas com as quais as crianças cotidianamente se envolvem em relações práticas (ou seja, em práticas) com outros grupos geracionais (Alanen, 200 la). Busca-se identificar as práticas geracionais por meio das quais as crianças se co "constroem" a si mesmas como "crianças", ou seja, como ocupantes de uma posição geracional particular em relação a um número de outros agentes (Alanen, 200 l , 200 la). Uma vantagem de estudar questões referentes às crianças [children's issues] de modo relacional é que elas ajudam a produzir uma análise mais dinâmica do que a abordagem categorial. Uma segunda vantagem é que não só os resultados de processos geracionais determinados podem ser estudados em relação a características que as crianças apresentam, mas também os processos reais e as relações no interior das quais esses resultados são produzidos. Portanto, também o aspecto da ação (social) [agency] na atividade das crianças fica mais proeminente, já que as crianças são estudadas como coconstrutoras da sua objetividade e subjetividade, das suas condições estruturadas e estruturantes.

Na conferência dos estudos sobre o bem-estar e a infância, as questões básicas para o desenvolvimento dos estudos do bem-estar das crianças são:

- até que ponto as referências, conceituações e abordagens dos estudos de bem-estar atuais são capazes de incorporar as "novas" perspectivas introduzidas pelos Estudos sobre a Infância?

- até que ponto se trata realmente de construções "adultistas" que resistem a incluir crianças em suas estruturas ou, ao incluí-las, o fazem comprometendo uma melhor compreensão das crianças?

- essas interpretações podem ser ajustadas e reorientadas a fim de conter as perspectivas mais avançadas sobre a infância?

Essas questões impõem uma crítica aos referenciais das pesquisas do bem-estar e a desconstrução de suas maneiras de pensar, com vistas ao desenvolvimento dos estudos de bem-estar da criança. A abordagem sugere uma postura crítica em relação às noções correntemente usadas nas abordagens no campo do bem-estar: seu contexto, pontos de partida e pressupostos básicos sobre as crianças e a infância, família, cidadania, justiça etc. 
No projeto Cost, recursos materiais, sociais e culturais, como rendimentos e riqueza, espaço e tempo, foram considerados do ponto vista de seus constrangimentos, oportunidades e limitações para que as crianças exerçam sua ação social. Essa ação, conforme foi apresentado, é a maior preocupação da Sociologia da Infância. A experiência subjetiva de constrangimentos, oportunidades e limitações, bem como o uso ou não uso de recursos disponíveis, podem fazer parte, de maneira importante, das infâncias experimentadas e vividas, e parecem, portanto, poder indicar o bem-estar/o sentir-se bem das crianças. A Sociologia da Infância oferece claramente um referencial para os estudos sobre o bem-estar das crianças. $\bigcirc$ trabalho de Tess Ridge (2002) sobre a experiência das crianças com a pobreza demonstra o uso da abordagem para atingir a dimensão subjetiva, ativa e experiencial do bem-estar das crianças. A abordagem abre-se também para os referenciais de bem-estar que conceituam especificamente as dimensões culturais e experienciais (subjetivas) do bem-estar (Chamberlayne et al., 1999; William, Popay, Oakley, 1999).

A metodologia proposta no projeto Cost assume o pressuposto estrutural-categorial que as crianças formam uma categoria de pessoas estabelecida institucional e culturalmente. Nesses termos, o acesso de crianças e a utilização por elas de fontes de recursos valiosas e relevantes para o bem-estar podem ser descritos, analisados e comparados entre os países. Essa abordagem combina, no mínimo, com aquela dos estudos de indicadores sociais, de qualidade de vida e com os trabalhos estatísticos sobre as várias "dimensões de bem-estar". Para informar uma sociologia do bem-estar das crianças, a abordagem estrutural-categorial deve enriquecer essas descrições analíticas aliando-as à argumentação relativa aos contextos estruturais que parecem produzir o fenômeno observado. As teorizações estruturais-categoriais podem, por sua vez, ser enriquecidas e desenvolvidas na direção de teorias mais dinâmicas e relacionais do bem-estar das crianças. Isso exige a observação e medição - e não somente a teorização abstrata - de realidades sistêmicas e dinâmicas, como as estruturas e os processos geracionais, bem como dos processos pelos quais os significados de bem-estar são produzidos e aplicados.

Em suma, a proposta para avançar na teorização sobre o bem-estar das crianças é que comecemos a integrar a pesquisa sobre bem-estar e o estudo sociológico da infância: 
- submetendo referenciais de pesquisa sobre bem-estar, paradigmas e abordagens existentes e suas definições normativas de bem-estar à desconstrução crítica do ponto de vista da infância;

- empregando uma visão sistêmica, multinível e processual de bem-estar que ofereça apoio às divisões, relações e posições geracionais nessa conceituação;

- utilizando as ferramentas analíticas sugeridas por uma perspectiva informada do ponto de vista estrutural e relacional sobre a infância para repensar e reorientar os recursos conceituais da pesquisa sobre bem-estar com vistas à incorporação das crianças e da infância.

Essa perspectiva de teorização impõe um pensamento analítico de tipo "móvel". Pensar o bem-estar das crianças será também, portanto, algo sempre inacabado e nos orientará "em direção à tentativa de entender o presente como uma conjuntura das rotas complexas e múltipas que conduziram a esse ponto" (Clarke, 2004, p.4-5). A perspectiva e a ambição não podem, portanto, oferecer uma teoria sobre o bem-estar das crianças.

\section{REFERÊNCIAS BIBLIOGRÁFICAS}

ALANEN, L. Childhood as a generational condition: children's daily lives in a central Finland town. In: ALANEN, L.; MAYALL, B. (Ed.). Conceptualizing child-adult relations. London: Routledge, 200I. p. I29-I 43.

. Explorations in generational analysis. In: ALANEN, L.; MAYALL, B. (Ed.). Conceptualizing child-adult relations. London: Routledge, 200 I a. p. I I-22.

. Modern childhood? Exploring the "child question" in sociology. Jyväskylä: Institute for Educational Research, University of Jyväskylä, 1992. (Series A: Research reports, 50) BARRY, N. Welfare. Buckingham: Open University, 1999.

BEAUVAIS, C.; JENSON, J. Two policy paradigms: family responsibility and investing in children. Otawa: CPRN, 200 I . (Discussion paper, n. F/I2). Disponível em: <http://www.cpm.org > . Acesso em: maio 2006.

BEN-ARIEH, A.; WINTERSBERGER, H. (Ed.). Monitoring and measuring the state of children: beyond survival. Vienna: European Centre, 1997. (Eurosocial reports, 62) 
BERGER-SCHMITT, R. Social cohesion as an aspect of the quality of societies: concept and measurement. Mannheim: Centre for Survey Research and Methodology, 2000. (Eureporting working paper, n. 14)

BERGER-SCHMITT, R.; NOLL, H.-H. Conceptual framework and structure of a European system of social indicators. Mannheim: Centre for Survey Research and Methodology, 2000. (Eureporting working paper, n. 9)

BILTON, K. Child welfare: whose responsibility? In: STEVENSON, O. (Ed.). Child welfare in the UK. Oxford: Blackwell, 1999. p.22-41.

$\mathrm{BLOCH}, \mathrm{M}$., et. al. Global and local patterns of governing the child, family, their care, and education. In: BLOCH, M. et al. (Ed.). Governing children, families and education. New York: Palgrave Macmillan, 2003. p.3-31.

BONOLI, G.; GEORGE, V. TAYLOR-GOOBY, P. European futures. Cambridge: Polity, 2000.

CARRINGTON, K. The Welfare/justice nexus. In: MASON, J. (Ed.). Child welfare policy: critical Australian perspectives. Sydney: Iremonger, 1993. p.69-88.

CHAMBERLAYNE, P. et al. (Ed.). Welfare and culture in Europe: towards a new paradigm in social policy. London: Jessica Kingsley, 1999.

CLARKE, J. Changing welfare, changing states: new directions in social policy. London: Sage, 2004.

CLARK, D. A.; GOUGH, I. Capabilities, needs and wellbeing: relating the universal and the local. In: MANDERSON. L. (Ed.). Rethinking well-being. Perth: Australian Research Institute, 2005. p.69-90.

CORBETT, TH. J. Foreword. In: HAUSER, R. M.; BROWN, B. V.; PROSSER, W. R. (Ed.). Indicators of children's well-being. New York: Russell Sage Foundation, 1997. p. 19-2I.

CURRIE, J. M. Welfare and the well-being of children. Chur: Harwood Academic, 1995.

ESPING-ANDERSEN, G. A child-centred social investment strategy. In: ESPINGANDERSEN, G. et al. (Ed.). Why we need a new welfare state. New York: Oxford University, 2002. p.26-67.

. The Three worlds of welfare capitalism. Cambridge: Polity, 1990.

EVERS, A.; OLK, T. (Hrsg.). Wohlfahrtspluralismus: Vom Wohlfahrtsstaat zur Wohlfahrtsgesellschaft. Opladen: Westdeutscher Verlag, 1996.

FALKINGHAM, J. The Impact of economic change on child welfare in Central Asia. In: VLEMINCKX, K.; SMEEDING, T. M. (Ed.). Child well-being, child poverty and child policy in modern nations: what do we know? Bristol: The Policy, 200 I . p.227-254. 
FINK, J. Silence, absence and elision in analyses of "the family" in European social policy. In: FINK, J.; LEWIS, G.; CLARKE, J. (Ed.). Rethinking European welfare. London: Sage/The Open University, 200 I. p. 163-180.

FINK, J.; LEWIS, G.; CLARKE, J. (Ed.). Rethinking European welfare. London: Sage, The Open University, 2001.

FROST, N.; STEIN, M. The Politics of child welfare. Hemel Hempstead: Harvester Wheatsheaf, 1989.

GASPER, D. Human well-being: concepts and conceptualizations. Helsinki: United Nations University, 2004 (Discussion paper, n.6)

GIULLARI, S. Welfare quadrangles and the moral character of increasingly elective social ties of support. In: CONFERENCE WELFARE AND THE SOCIAL BOND, March 26-27 2003, Tilburg. Paper... Tilburg: University Tilburg, 2003. Disponível em: < http://spitswww.uvt.nl/ fsw/espanet/documents/doc/giul.doc >. Acesso em: maio 2006.

GOLDSON, B.; LAVALETTE, M.; MCKECHNIE, J. Children, welfare and the State. London: Sage, 2002.

GOUGH, I. Lists and thresholds: comparing the Doyal-Gough theory of human need with Nussbaum's capabilities approach. Bath: ESRC Research Group on Wellbeing in Developing Countries, 2003. (Working paper, n. I)

HARDING, S. The Science question in feminism. Ithaca: University, 1986.

HAUSER, R. M.; BROWN, B.V.; PROSSER, W. R. (Ed.). Indicators of children's well-being. NewYork: Russell Sage Foundation, 1997.

HENDRICK, H. Child welfare: historical dimensions, contemporary debate. Bristol: Policy, 2003. . (Ed.). Child welfare and social policy. Bristol: Policy, 2005.

HENGST, H. Kinder und Ökonomie: Aspekte gegenwärtigen Wandels. In: KRÄNZL-HAGL, R.; MIERENDORFF, J.; OLK, T. (Ed.). Kindheit im Wohlfahrtsstaat: Gesellschaftliche und politische Herausforderungen. Frankfurt: Europäisches Zentrum; New York: Campus, 2003. p.235-266.

HUDSON, J.; LOWE, S. Understanding the policy process. Bristol: Policy, 2004.

JAMES, A.; JENKS, C.; PROUT, A. Theorizing childhood. Cambridge: Polity, 1998.

JENSEN, A.-M. et al. (Ed.). Children's welfare in ageing Europe. Trondheim: Norwegian Centre for Child Research, Norwegian University of Science and Technology, 2004. v. I/2. 
JENSON, J. Changing the paradigm: family responsibility or investing in children. Canadian Journal of Sociology, Peterborough, v. 29, n.2, p. 169-192, 2004.

JOOS, M. Die Soziale Lage der Kinder: Sozialberichterstattung über die Lebensverhältnisse von Kindern in Deutschland. Weinheim \& München: Juventa, 2001 .

KRÄNZL-NAGL, R.; MIERENDORFF, J.; OLK, T. (Ed.). Kindheit im Wohlfahrtsstaat: Gesellschaftliche und politische Herausforderungen. Frankfurt: Europäisches Zentrum; New York: Campus, 2003.

LADERCHI, C. R.; SAITH, R.; STEWART, F. Does it matter that we don't agree on the definitions of poverty? A comparison of four approaches. Oxford: University of Oxford, 2003. (QEH working paper series, n. 107). Disponível em: <http://www2.qeh.ox.ac.uk/pdf/qehwp/ qehwps 107.pdf>. Acesso em: maio 2006.

LISTER, R. Investing in the citizen of the future: new labour's "third way" in welfare reform. In: ANNUAL MEETING OF AMERICAN POLITICAL ASSOCIATION, 2002. Paper presented... 2002. Disponível em: <http://www.fas.umontreal.ca/pol/cohesionsociale/publications/ister. pdf >. Acesso em: maio 2006. . Poverty. Cambridge: Polity, 2004.

MANDERSON, L. (Ed.). Rethinking wellbeing. Perth: Australia Research Institute, Curtin University of Technology, 2005.

MEMORANDUM OF UNDERSTANDING. Cost AI 9 children's welfare, 2000. Disponível em: <http://www.svt.ntnu.no/noseb/costal9/>. Acesso em: maio 2006.

MICKLEWRIGHT, J.; STEWART, K. The Welfare of Europe's children. Bristol: Unicef, Policy, 2000.

O'BRIEN, M.; PENNA, S. Theorising welfare: enlightenment and modern society. London: Sage, 1998.

PIERSON, C. Beyond the welfare state. Cambridge: Cambridge University, 1998.

QVORTRUP, J. Children at risk or childhood at risk: a plea for a politics of childhood. In: HEILIÖ, P.-L.; LAURONEN, E.; BARDY, M. (Ed.). Politics of childhood and children at risk. Vienna: European Centre, 1993. p. 19-30. (Eurosocial report, n. 45)

RAPLEY, M. Quality of life research: a critical introduction. London: Sage, 2003.

RIDGE, T. Childhood poverty and social exclusion, from a child's perspective. Bristol: Policy, 2002. STEVENSON, O. (Ed.). Child welfare in the UK. Oxford: Blackwell Science, 1999. 
TITMUSS, R. The Relationship between income maintenance and social service benefits: an overview. International Social Security Review, Geneva, v.20, n. I, p.57-66, 1967.

TRIFILETTI, R. Southern European welfare regimes and the worsening position of women. Journal of European Social Policy, Edinburgh, v. 9, n. I, p.49-64, 1999.

VLEMINCKX, K.; SMEEDING, T. M. (Ed.). Child well-being, child poverty and child policy in modern nations: what do we know? Bristol: Policy, 200I.

WILLIAM, F.; POPAY, J.; OAKLEY, A. Welfare research: a critical review. London: UCL, 1999.

Recebido em: agosto 2010

Aprovado para publicação: agosto 2010 